\title{
PET imaging of glucose and fatty acid metabolism for NAFLD patients
}

\author{
Grant T. Gullberg, PhD, ${ }^{\mathrm{a}}$ Uttam M. Shrestha, PhD, ${ }^{\mathrm{a}}$ and Youngho Seo, $\mathrm{PhD}{ }^{\mathrm{a}}$ \\ a Department of Radiology and Biomedical Imaging, University of California, San Francisco, CA
}

Received Nov 8, 2018; accepted Nov 9, 2018

doi: $10.1007 / \mathrm{s} 12350-018-01532-8$

\section{See related article, pp. 1679-1688}

The paper presented in this issue of the Journal by Tang and colleagues illuminate the deficiency in our knowledge of the reciprocal metabolic relationship between liver and heart in health and disease. ${ }^{1}$ The results presented in this paper used a large number of previously acquired ${ }^{18} \mathrm{~F}$-FDG PET studies to investigate the association between non-alcoholic fatty liver disease (NAFLD) (Figure 1A) and myocardial glucose uptake. Previous work published by Lee and colleagues in Metabolism, ${ }^{2}$ had found a significant correlation between NAFLD and vascular inflammation using ${ }^{18} \mathrm{~F}$ FDG PET to measure maximum target-to-background uptake in the carotid arteries; however, the present study is the first to elicit through imaging the correlation between NAFLD and potential cardiac metabolic abnormalities. Patients with NAFLD have a high risk of related cardiovascular disease $(\mathrm{CVD}){ }^{3}$ It has been pointed out that the leading cause of death in NAFLD patients is CVD rather than liver-associated complications, ${ }^{4}$ of which only $5 \%$ of NAFLD patients die from liver-related diseases. ${ }^{5}{ }^{18} \mathrm{~F}$-FGD PET is an important indicator of cardiac glucose metabolism and its alteration in the presence of disease; however, to systematically understand the relation between NAFLD and the risk of cardiovascular disease, other probes, in addition to ${ }^{18} \mathrm{FDG}$, should be used to study the complex and dynamic pathways of energy substrate metabolism in the heart in health and disease and their relationship to the metabolic pathways of other body organs.

Reprint requests: Grant T. Gullberg, $\mathrm{PhD}$, Department of Radiology and Biomedical Imaging, University of California, San Francisco, CA; gtgullberg@lbl.gov

J Nucl Cardiol 2020;27:1689-97.

$1071-3581 / \$ 34.00$

Copyright (C) 2018 American Society of Nuclear Cardiology.
The aim of the paper by Tang and colleagues was to investigate the association between NAFLD and myocardial glucose uptake to see if alterations in ${ }^{18} \mathrm{~F}$ FDG uptake could be an indicator of cardiac dysfunction in NAFLD individuals. They retrospectively assessed ${ }^{18}$ F-FDG PET imaging data of a total of 201 subjects with NAFLD and 542 without NAFLD who were imaged over the years from December 1, 2011 to November 30, 2017. The liver (Figure 1B) in addition to adipose tissue is a major source of lipoproteins (Figure 1C), which are a major substrate for cardiac ATP production. NAFLD is a disease with an extensive amount of fat in the liver. The diagnosis of fatty liver disease was confirmed by CT where disease was indicated if liver attenuation was at least 1 Hounsfield Unit (HU) less than the spleen and the attenuation ratio of liver to spleen was less than 1.0. It was found that myocardial ${ }^{18}$ F-FDG uptake was significantly lower in individuals with NAFLD compared with those without fatty liver. The authors also demonstrated that in NAFLD patients the glucose uptake was inversely proportional to the increase in left ventricular (LV) filling pressure, suggesting that NAFLD individuals with lower myocardial ${ }^{18} \mathrm{~F}$-FDG uptake are more likely to have high risk of impaired diastolic heart function. The reasons for alterations of the biochemical processes of myocardial glucose uptake in patients with NAFLD is not clear, but probably is the result of several complex biochemical processes involving genetic factors, atherosclerosis, inflammatory cytokines, insulin resistance, and other alterations in biochemistry of glucose and fatty acid metabolism. 6,7

The process of glucose and fatty acid metabolism converts chemical energy into mechanical energy that is regulated by hemodynamic factors, neurohumoral factors, and oxygen availability. ${ }^{8,9}$ It involves translocation of molecular substrates into the cell and mitochondria, the facilitated diffusion of molecular species, enzymatic processes in metabolic pathways, and genetic regulation of enzyme production, where many things can go wrong. 
In the heart, energy production in the form of ATP involves the metabolism of $70 \%$ to $80 \%$ fatty acids and $20 \%$ to $30 \%$ glucose (glucose, lactate, glycogen). ${ }^{10}$ The ATP produced is used in several chemical reactions involving sequestering of substrates into the cell, transport of ions (such as $\mathrm{Ca}^{++}$) in and out of internal vesicles and ions (such as $\mathrm{Na}^{+}, \mathrm{K}^{+}$) in and out of myocytes during nerve impulse propagation, breaking down of metabolic substrates (chemical synthesis), and the production of mechanical translation between actin and myosin fibers (muscle contraction). The heart turns over its ATP pool every 10 seconds. ${ }^{10}$ Metabolism of carbohydrates produces $4 \mathrm{kcal} / \mathrm{gram}$ of energy, whereas metabolism of fatty acids produces $9 \mathrm{kcal} / \mathrm{gram}$. The use of fatty acids as a substrate generates the greater number of ATP, but it comes at the expense of a greater oxygen requirement than the use of glucose. Because of the many chemical processes and transport of molecular species, the conversion of chemical energy into mechanical energy is only approximately $20 \%$ efficient. $^{10}$

The significance of understanding cardiac metabolism of fuels, such as glucose and fatty acids, is now appreciated in heart disease. ${ }^{10,11}$ Glucose metabolism begins with the transport of glucose into the cell. This is facilitated by GLUT transporters of which GLUT-1 (insulin insensitive) and GLUT-4 (insulin sensitive) are the major glucose transporters. ${ }^{8}$ When insulin binds to receptors on the surface of a cell (Figure 2A), this signals a cascade of reactions activated by phosphatidylinositol kinase activity which results in the movement of cytoplasmic vesicles containing GLUT-4 toward the cell surface. The vesicles fuse with the cell surface membrane embedding the GLUT-4 protein channels into the membrane. In the cell cytoplasm, glucose is initially converted to glucose 6-phosphate by the enzyme hexokinase. In PET imaging ${ }^{18}$ FDG undergoes this first step to ${ }^{18}$ FDG-6-phosphate but then is unable to continue through glycolysis. G6P is a branching point in carbohydrate metabolism: ${ }^{8}$ (1) G6P can serve as a precursor for glycogen (Figure 2B) synthesis; (2) G6P can proceed into glycolysis after being committed by PFK-1; (3) G6P can enter the pentose phosphate pathway; or 4) G6P can be a precursor of uridine diphosphate-Nacetylglucosamine (UDPGlcNAc). The first step in committing glucose to the glycolytic pathway is the phosphorylation of fructose-6phosphate to fructose 1,6-bisphosphate by the enzyme 6phospho-fructo-1-kinase (PFK-1). This is a pacemaker enzyme for glycolysis. ${ }^{8,9}$ From here fructose 1,6-bisphosphate continues down the glycolytic pathway to pyruvate. Glycolysis reduces a 6-carbon glucose molecule down to two 3-carbon pyruvate molecules. In the process ATP is made independent of oxygen through an anaerobic process. The entire process results in the production without oxygen of two ATP, two NADH,

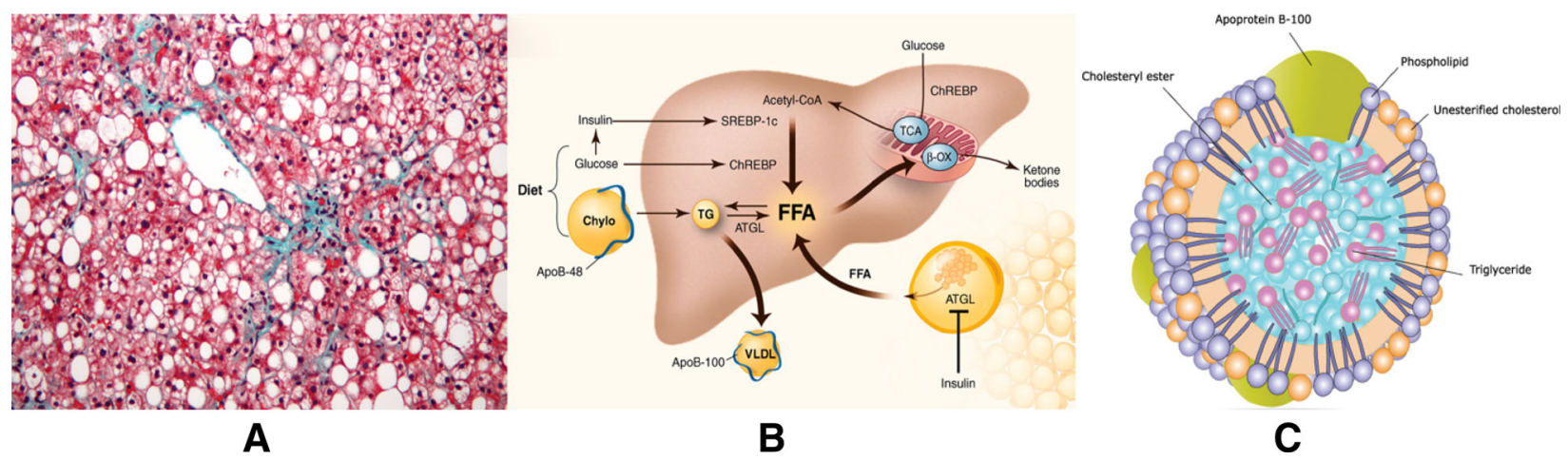

Figure 1. A Non-alcoholic fatty liver disease (NAFLD) is the excessive accumulation of fat in the liver of people who consume very little or no alcohol. Fat deposits (white/clear round/oval spaces) and mild fibrosis (green) are shown in a micrograph tissue sample of nonalcoholic fatty liver disease. The tissue is stained with Masson's trichrome \& Verhoeff stain. The hepatocytes stain red. Figure used under a Creative Commons Attribution-ShareAlike 3.0 Unported license (https://c ommons.wikimedia.org/wiki/File:Non-alcoholic_fatty_liver_disease1.jpg). B Liver has many functions in the body, including a major synthesize of very low density lipoproteins (VLDL) for cardiac lipoprotein-derived fatty acids. The ultra low-density lipoproteins (ULDL) called chylomicrons are from dietary fat. Adipose triglyceride lipase may play an important role in developing NAFLD. This figure was originally in Science 2011;332:1519, and reprinted with permission from AAAS. C Lipoprotein shown here as a micelle with a polar surface of phospholipid monolayer, free cholesterol, and apolipoproteins. The non-polar lipid core contains cholesterol esters, and triacylglycerides. Reprinted with permission from ShutterStock (ID: 246595399). 


\section{Effect of Insulin on Glucose Uptake}

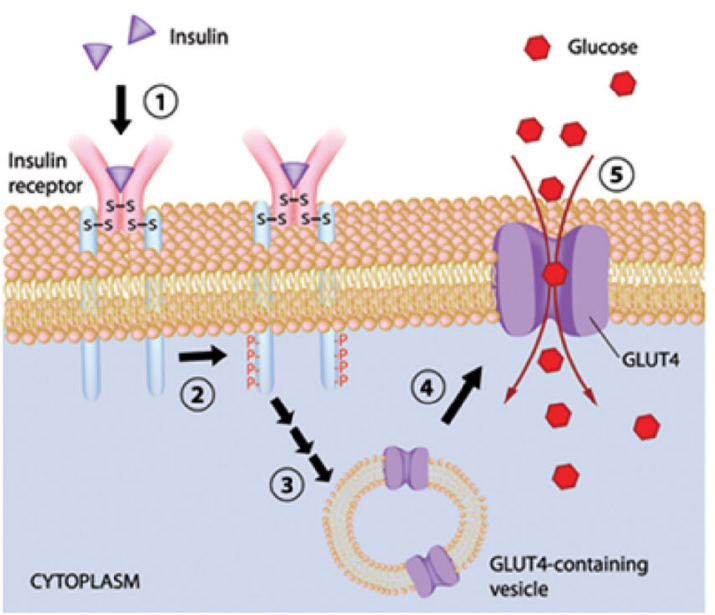

A

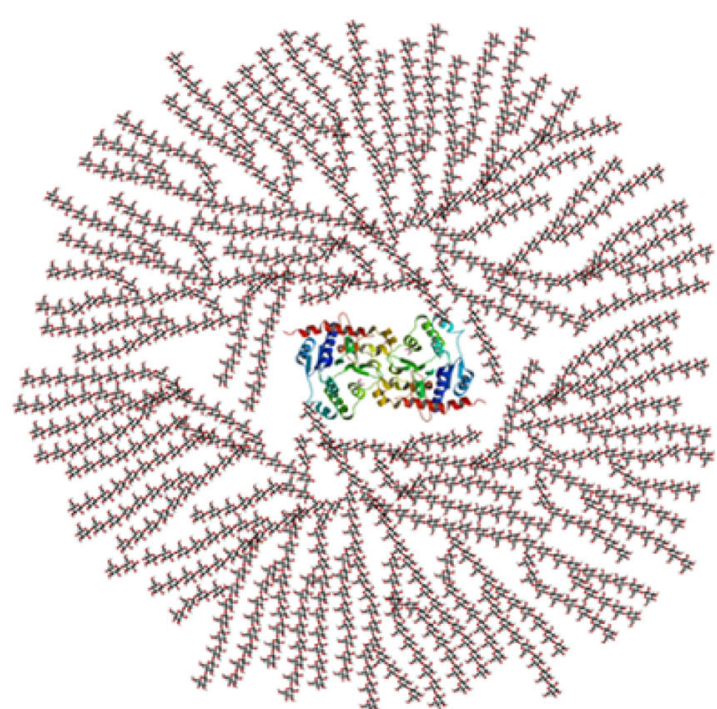

B

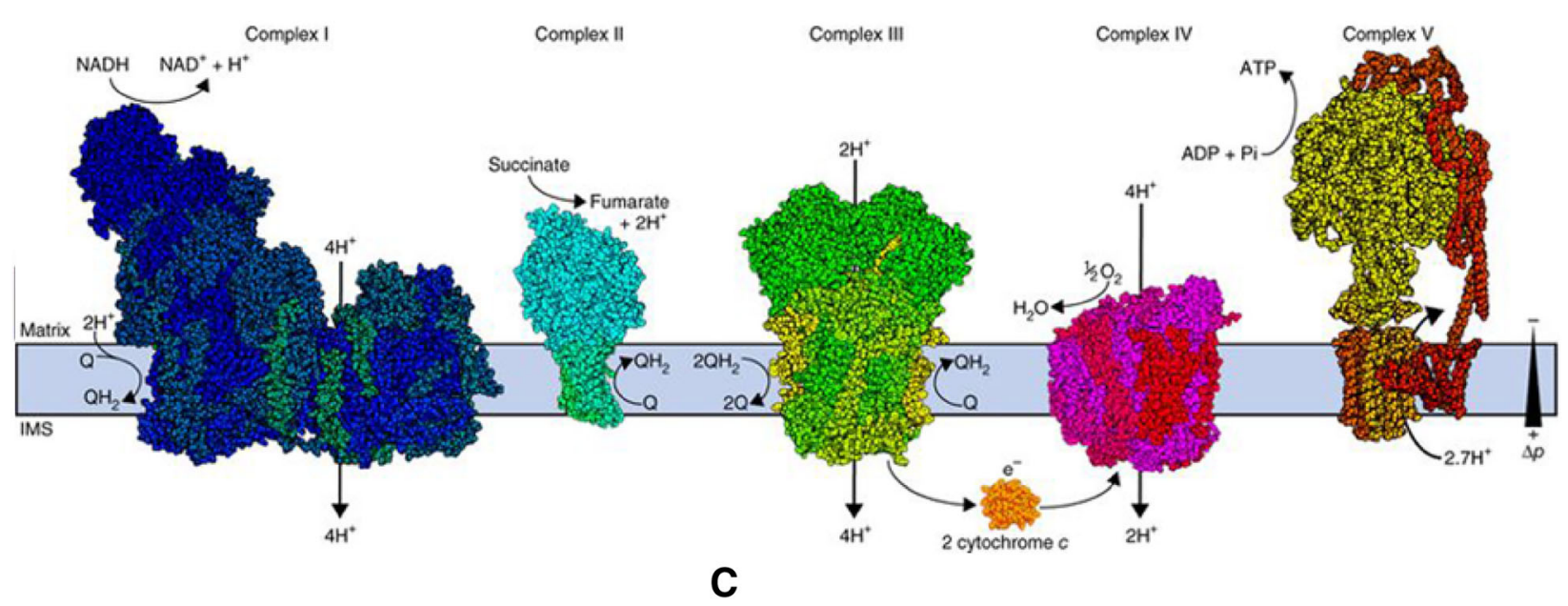

Figure 2. A Insulin binds to its receptor, which starts cascades of biochemical events including translocation of Glut-4 transporter to the plasma membrane and influx of glucose. Reprinted with permission from ShutterStock (ID:114645271). B Glycogen is the main storage of glucose in myocytes and is shown here in a two-dimensional cross-sectional contains a core protein of glycogen in surrounded by branches of glucose units (as many as 30,000). Public domain image (https://commons.wikimedia.org/wiki/File:Glycogen_structure.svg). C Electron transport chain of oxidative phosphorylation. The oxidation of NADH releases an electron which is transferred along the electron chain between protein complexes (Complex I, III, and IV) extruding $\mathrm{H}^{+}$ions across the inner mitochondrial membrane into the space between the inner and outer mitochondrial membranes. The oxidation of $\mathrm{FADH}_{2}$ results in transferring of an electron between Complex II and III with the extrusion of $\mathrm{H}^{+}$ions across the inner mitochondrial membrane. This produces ATP as $\mathrm{H}^{+}$ions are transported back into the mitochondria by way of transport with ATP synthases converting ADP to ATP with the production of water. Reprinted from Nat Struct Mol Biol. 2017;24:800, by permission from Springer Nature.

and two 3-carbon molecules of pyruvate. The pyruvate molecule is either converted to lactate or is actively transported into the mitochondria by pyruvate translocase. The pyruvate is then converted to acetyl-co- enzyme $\mathrm{A}(\mathrm{CoA})$ plus $\mathrm{CO}_{2}$ and NADH. The acetyl-CoA is the primary input into the Krebs cycle to complete the aerobic metabolism. Pyruvate can also be converted to oxaloacetate to replenish Krebs cycle intermediates. The 
Krebs cycle produces the energetic enzymes NADH and $\mathrm{FADH}_{2}$ involved in producing ATP through oxidative phosphorylation (Figure 2C).

In the circulation, fatty acids are predominantly the long-chain fatty acids oleate and palmitate. ${ }^{9}$ The pathway of long-chain fatty acid oxidation starts in the circulation with the liberation from triglycerides and crossing the plasma membrane with the help of transporter proteins. There are three major fatty acid transporters in the heart of which the fatty acid translocase (FAT/CD36) (Figure 3A) is the major longchain fatty acid uptake regulator. ${ }^{12}$ After entering the cell, long-chain-fatty-acid-CoA synthetase (LC-FACS) catalyzes the reaction between a fatty acid molecule and ATP to give fatty acyl-adenylate that then reacts with the free coenzyme A (CoA) to give fatty acyl-CoA. The fatty acyl-CoA is then able to enter the mitochondrion by way of the carnitine shuttle (Figure 3B) with the assist of carnitine palmitoyltransferase I (CPT1) and carnitine palmitoyltransferase I (CPT2) and carnitineacyl-carnitine translocase (CAT). In the mitochondrial matrix $\beta$-oxidation cuts the long carbon chains of fatty acids into a series of two carbon acetate units. These acetate molecules combine with CoA to form acetylCoA. Each $\beta$-oxidative cut of the fatty acyl-CoA molecule yields a fatty acyl-CoA molecule of 2 less carbon plus an acetyl-CoA molecule and $1 \mathrm{NADH}$ and 1 $\mathrm{FADH}_{2}$ (producing 5 ATP molecules by oxidative phosphorylation). ${ }^{13}$ The acetyl-CoA enters the Krebs cycle by combining with oxaloacetate to form citrate at the beginning of the citric acid cycle. Each turn of the cycle results in the complete combustion of the acetyl$\mathrm{CoA}$ to $\mathrm{CO}_{2}$ and water and releases energy in the form of $1 \mathrm{GDP}$ and $3 \mathrm{NADH}$ molecules. At the site of oxidative phosphorylation on the inner membrane of the mitochondria, these reducing agents release electrons to the electron transport chain of protein complexes (Figure $2 \mathrm{C}$ ). This results in an energy conversion by oxidative phosphorylation of 11 ATP molecules per acetyl-CoA molecule oxidized. ${ }^{13}$ The coenzyme ubiquinone (coenzyme $\mathrm{Q}_{10}$ ) is important as an electron carrier in the inner membrane of the mitochondria. Reduction of ubiquinone to ubiquinol $\left(\mathrm{QH}_{2}\right)$ by electron transport contributes to the generation of the proton gradient. Cardiolipin (CL) is a diphosphatidylglycerol lipid that constitutes about $20 \%$ of the total lipid composition of the inner mitochondrial membrane. During oxidative phosphorylation large quantities of protons are transferred from the inner mitochondrial membrane into the space between the inner and outer mitochondrial membranes causing a large $\mathrm{pH}$ change. It is suggested that $\mathrm{CL}$ functions as a proton trap within the

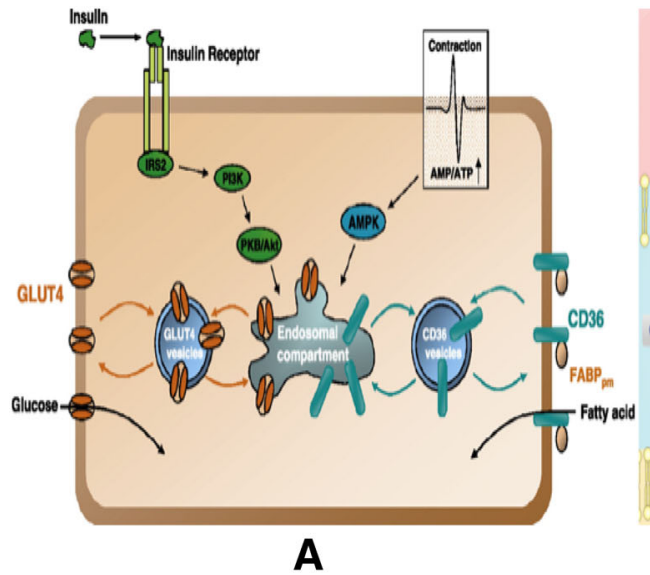

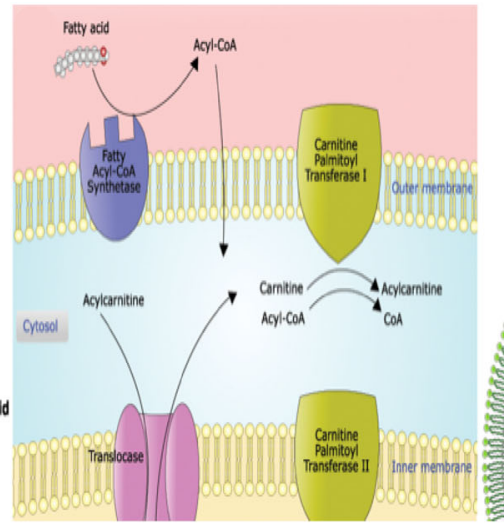

B

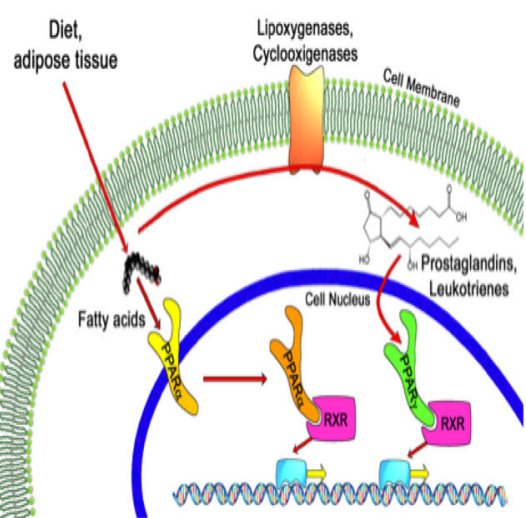

C

Figure 3. A Insulin and contraction stimulates translocation of glucose and fatty acid cell membrane transporters. Reprinted from Prostaglandins, Leukotrienes and Essential Fatty Acids. 2010;82:149, with permission from Elsevier. B Carnitine shuttle transports fatty acids between cytoplasm and mitochondrion. The fatty acyl-CoA first combines with carnitine to form acylcarnitine and eliminating CoA with the assist of CPT1. The fatty acyl-carnitine crosses the external mitochondrial membrane. Then the fatty acyl-carnitine crosses the internal mitochondrial membrane via CAT through facilitated diffusion. Carnitine is then exchanged for CoA by CPT2 to the original fatty acyl-CoA. The released carnitine diffuses back across the membrane by CAT into the mitochondrial intermembrane space. Reprinted with permission from ShutterStock (ID: 279851630). C Peroxisome proliferator-activated receptors (PPARs) regulate synthesis of enzymes for fatty acid metabolism by providing a high degree of transcriptional control of gene expression. Figure from https://upload.wikimedia.org/wikipedia/commons/4/43/PPAR-diagram.pn $\mathrm{g}$, used under Creative Commons CC0 1.0 Universal Public Domain Dedication. 
mitochondrial membranes by localizing the proton pool and minimizing changes in $\mathrm{pH}$ in the mitochondrial inter-membrane space.

Abnormal structure and function of liver cells in NADFL patients may provide insight into cardiac cellular abnormalities. The liver (Figure 1B) is the central organ for handling lipids including storage of glycerols and fats in its hepatocytes, manufacturing triglycerides and cholesterol, synthesizing glycogen, and producing bile from cholesterol. It is also involved in making proteins and blood clotting factors. Another important organ closely related to the liver for manufacturing and storage of energetic substrates is adipose tissue whose main role is to store energy in the form of lipids, where there is a constant flux of free fatty acids entering and leaving its cellular adipocytes. Free fatty acids are liberated from lipoproteins (Figure 1C) by lipoprotein lipase (LPL) and enter the adipocyte, where they are reassembled into triglycerides by esterifying it onto glycerol. The net direction of this flux is controlled by insulin and leptin and only when insulin is low can free fatty acids leave adipose tissue.

It is recognized that cardiac cells differ in many respects to liver cells. Cardiac cells need continuous energy supply but have poor capacity to synthesize and store energy substrates, whereas liver cells can synthesize, store, and release energy substrates. However, they have similarities and understanding irregularities in liver cells of NAFLD patients may give insight into potential defects in cardiac cells. In NAFLD patients, an increase in hepatic levels of triacylglycerol (TG), diacylglycerol (DG), cholesteryl ester (CE), and free cholesterol and a change in the percent of polyunsaturated fatty acid (PUFA) in these lipids has been observed. As indicated in an excellent review by Peng and colleagues, ${ }^{14}$ this may be due to mitochondrial abnormalities found in NAFLD patients indicating metabolic alterations. These abnormalities are seen as abnormal morphological changes, leaky mitochondrial membranes, accumulation of structural and enzymatic proteins, and oxidative stress. Mitochondrial molecular components of cardiolipin, ubiquinone, and acyl-carnitine play an important role in mitochondrial function of oxidative phosphorylation. It is suggested that cardiolipin and ubiquinone levels increase in NAFLD to enhance mitochondrial respiration, thus reducing steatosis (abnormal retention of lipids). On the other hand, acyl-carnitine involved in the carnitine shuttle (Figure 3B) increases with NAFLD indicative of mitochondrial stress, mitochondrial dysfunction, and impaired fatty acid oxidation. Modulation of action of enzymatic proteins through allosteric regulation may also change the mix of metabolic substrates with the disease. For example, methylmalonate semialdehyde dehydrogenase and propionyl-CoA carboxylase are involved in the metabolism of succinylCoA (component of the tricarboxylic acid cycle). It is known that these enzymes, which are decreased in mitochondria of obese individuals, could also be decreased in NAFLD patients. In addition, there is evidence that increased dihydroceramide and dihexosylceramides with NAFLD may increase metabolism of ceramides and contribute to metabolic disorders in the disease. ${ }^{14}$ Ceramides (composed of sphingosine and a fatty acid) are found in high concentrations within the cell membrane providing supportive function and also cellular signaling, including regulating differentiation, proliferation, and programmed cell death.

Decreased glucose metabolism may be a potential indication of cardiac lipotoxicity in NAFLD patients. Cardiac lipotoxicity (Wan and Rodrigues, ${ }^{8}$ Schulze et al. $^{15}$ ) is the excessive accumulation of intramyocellular fatty acids and their metabolites, and is a main contributor to the pathophysiology of insulin resistance and dysfunctioning of heart muscle. ${ }^{16}$ Fatty acids are delivered to the heart by (1) lipolysis in adipose tissue releasing fatty acid into the plasma, (2) LPL mediated breakdown of TG-rich lipoproteins from the liver (VLDL) and gut (chylomicron), and (3) endogenous triglyceride (TG) breakdown within the heart. The paper of Tang and colleagues in this issue found higher levels of fatty acids in the blood of NAFLD patients. Arterial fatty acid concentration is the primary determination of the rate of myocardial fatty acid uptake and oxidation, and intramyocardial triacylglycerol (TAG) content. $^{12}$ The transport of fatty acids into the cell is facilitated by FAT/CD transporters. Both muscle contraction and insulin (Figure 3A) stimulate the translocation of FAT/ CD36 to the cell membrane, which is mediated by 5' adenosine monophosphate-activated protein kinase (AMPK). This translocation occurs independently of the insulin-induced translocation mediated by phosphatidylinositol 3-kinase (PI3K). ${ }^{16}$ It has been shown that increased CD36 abundance in the cell membrane and an increased plasma fatty acid concentration not only can lead to an excessive increase in fatty acid uptake and oxidation but also leads to an increased rate of fatty acid esterification into TAG and increased concentrations of fatty acid metabolites of diacylglycerols (DAG) and ceramides. These intermediates of TAG, DAG, and ceramides are potentially harmful intracellular components that can create insulin resistance by reducing the insulin-induced GLUT4 translocation to the sarcolemma and lowering the rate of glucose uptake. ${ }^{16}$

There are many feedback controls between glucose and fatty acid metabolism. For one, the oxidation of pyruvate and the activity of pyruvate dehydrogenase 
(PDH) in the heart are decreased by elevated rates of fatty acid $\beta$-oxidation; whereas, pyruvate oxidation is enhanced by suppression of fatty acid $\beta$-oxidation. The enzyme AMPK is particularly important as a fuel sensor that plays a big role in cellular energy homeostasis to increases fatty acid uptake and increase $\beta$-oxidation during times of increased energy demand or decrease fatty acid uptake and $\beta$-oxidation in times of low demand. ${ }^{12}$ For example, through the AMPK-ACC-MCD axis the concentration of malonyl-CoA depends on the balance between acetyl-CoA carboxylase (ACC) and malonyl-CoA decarboxylase (MCD). Other enzymes involved in the fatty acid $\beta$-oxidation are sensitive to feedback inhibition through allosteric control by the products of enzymatic reactions, including $\mathrm{FADH}_{2}$ and NADH. These enzymes are also under a high degree of transcriptional control by peroxisome proliferator-activated receptors (PPARs) that regulate the expression of genes (Figure 3C). For one, the nuclear receptor PPAR $\alpha$ is a major transcriptional regulator of fatty acid metabolism whereby overexpression of $\operatorname{PPAR} \alpha$ results in increase in cardiac fatty acid uptake, fatty acid $\beta$-oxidation, and lipid overload; and under expression of PPAR $\alpha$ results in decreased expression of fatty acid $\beta$ oxidation and a parallel increase in glucose oxidation.

There are several other biological components involved in controlling the homeostasis of energy production. For example, phospholipids in cell membranes are involved in signaling to activate metabolic processes. In the membrane of the mitochondrion there are uncoupling proteins that are transport proteins providing an alternate means for the re-entry of protons from the inter-membrane space to the mitochondrion matrix that is not coupled to synthesis of ATP. These help maintain concentration and electrostatic balance between the inner mitochondrial membrane and the space between the inner and outer mitochondrial membrane. In disease these concentrations can become out of balance. It has been shown that enzymatic activities of mitochondrial complex I and II are significantly reduced in 24 weekold spontaneously hypertensive rats (SHR). ${ }^{17}$ Recently, increased levels of branched-chain amino acid (BCAA) have also been reported in animal models of heart failure. ${ }^{18}$ The end products of catabolism of BCAAs can enter the Krebs cycle either for oxidative decarboxylation or anaplerosis (the formation of intermediates of a metabolic pathway). ${ }^{10}$ BCAAs, especially leucine, increase mammalian target of rapamycin (mTOR) activity and thereby promote protein synthesis, cellular metabolism, and cell growth. ${ }^{19}$

Advances in PET metabolic imaging provides a necessary tool to further our understanding of various forms of cardiovascular disease and potentially improve the care of cardiac patients. ${ }^{20}$ In particular, PET imaging of glucose metabolism, ${ }^{21}$ fatty acid metabolism, ${ }^{22}$ and oxygen utilization ${ }^{23,24}$ provides information about metabolic shifts related to cardiac function, ${ }^{25}$ making PET an outstanding tool to measure cardiac metabolic changes in patients with NAFLD. There have been some imaging studies investigating liver metabolism in NAFLD patients, one using ${ }^{11} \mathrm{C}$-palmitate ${ }^{26}$ and one using ${ }^{18} \mathrm{~F}-\mathrm{FDG}$ and ${ }^{18} \mathrm{~F}$-FTHA ${ }^{27}$ and another study of NAFLD patients using ${ }^{18}$ F-FDG to image vascular inflamation, ${ }^{2}$ but no studies to evaluate the disease relationship to cardiac metabolism other than the study presented in this issue of the Journal by Tang and colleagues using ${ }^{18} \mathrm{~F}$-FDG. Using other metabolic imaging tracers to measure myocardial fatty acid metabolism would help to better measure more precisely and understand the reason for the metabolic shifts in this particular disease.

Most of our understanding of shifts in metabolic substrates in the heart has been elicited from PET imaging studies in heart failure. ${ }^{10,11,28-32}$ Using ${ }^{15} \mathrm{O}$ labeled water and ${ }^{11} \mathrm{C}$-labeled acetate, palmitate, and glucose tracers, one can assess simultaneously myocardial blood flow, energy expenditure, and fatty acid and glucose metabolism. ${ }^{28}$ Studies have shown decreased fatty acid utilization and increased myocardial glucose metabolism in patients with idiopathic dilated cardiomyopathy. ${ }^{28}$ On the other hand, patients with congestive heart failure, myocardial uptake of a radiolabeled fatty acid analog $\left({ }^{18}\right.$ F-FTHA) was greater and radiolabeled deoxyglucose $\left({ }^{18} \mathrm{~F}-\mathrm{FDG}\right)$ uptake was less than controls. ${ }^{31}$ When patients in class III HF were treated with carvediol, myocardial uptake of ${ }^{18} \mathrm{~F}$-FTHA decreased and ${ }^{18}$ F-FDG uptake was unchanged. ${ }^{32}$ The discrepancy among these investigations may be attributable to the severity of $\mathrm{HF}$, where in the early stages there is a normal (or slightly elevated) rate of fatty acid oxidation, with a downregulation in advanced stage of HF. ${ }^{10}$ In all of our imaging studies ${ }^{33,34}$ glucose metabolism was consistently elevated in the hypertensive SHRs compared to the normotensive WKY controls. When fed, the fatty acid metabolism in the SHRs was less than with the WKY controls. ${ }^{33}$ Whereas with fasting, there was an observed increase (though not significant) in fatty acid metabolism in the SHR model compared with WKY controls. ${ }^{34}$ Metabolic changes appear to precede mechanical changes of LVH progression in the SHR model.

A recent paper in this journal by Manabe and colleagues $^{35}$ gives an extensive review of possible PET cardiac tracers to evaluate cardiovascular disease by targeting myocardial perfusion, metabolism, innervation, and inflammation. Another interesting paper by $\mathrm{Li}$ and colleagues ${ }^{36}$ highlights efforts to develop tracers that target mitochondrion, which plays a fundamental 
role in cellular processes ranging from metabolism to apoptosis. Although perfusion is important, PET imaging of cardiac metabolism in heart failure continues to be the main focus in the development of new tracers. ${ }^{37}$ Another important application is the targeting of the development of fibrosis, especially important in hypertensive cardiac myopathy. Potentially using ${ }^{11} \mathrm{C}$ martinostat, which binds with high affinity to class 1 and 2 histone deacetylases (HDACs), ${ }^{38}$ would help to facilitate the development of novel anti-fibrotic therapies to reduce fibrosis in many heart failure patients.

A paper by $\mathrm{Wu}$ and colleagues ${ }^{39}$ provides insights into the design of PET fatty acid tracers. $\left[{ }^{11} \mathrm{C}\right]$-palmitate is a 16-carbon fatty acid commonly used for measuring myocardial fatty acid metabolism in PET studies, ${ }^{40}$ however, it is degraded rapidly within 2 minutes by complete $\beta$-oxidation in cardiac tissue. ${ }^{41}$ Therefore, effort has been made to develop tracers that are trapped in the cell by insertion of an S-atom or substitution with a methyl group preventing oxidative degradation. ${ }^{42}$ An example of a $\beta$-methyl modified fatty acid labeled with ${ }^{11} \mathrm{C}$ is $1-\left[{ }^{11} \mathrm{C}\right]-\beta$-R,S-methylheptadecanoic acid $\left(\left[{ }^{11} \mathrm{C}\right]-\right.$ BMHA). $\left[{ }^{11} \mathrm{C}\right]$-BMHA has high retention of activity in the myocardium and excellent imaging properties. ${ }^{41}$ Examples of fatty acids with an insertion of a S-atom include thia fatty acids labeled with ${ }^{18} \mathrm{~F}$ : $14-{ }^{18} \mathrm{~F}$-fluoro6-thia-heptadecanoic acid $\left(\left[{ }^{18} \mathrm{~F}\right]-\mathrm{FTHA}\right),{ }^{42} 18$ - $\left[{ }^{18} \mathrm{~F}\right] \mathrm{flu}$ oro-4-thia-oleate $\quad\left(\left[{ }^{18} \mathrm{~F}\right]-\mathrm{FTO}\right), \quad 16-\left[{ }^{18} \mathrm{~F}\right]$ fluoro-4-thiapalmitate $\left(\left[{ }^{18} \mathrm{~F}\right]\right.$-FTP $)$. These ${ }^{18} \mathrm{~F}$-labeled tracers demonstrate excellent properties as myocardial PET tracers. ${ }^{39,41}$

Metabolic therapies that stimulate myocardial carbohydrate oxidation and inhibit myocardial fatty acid oxidation can improve cardiac performance and prevent or reverse the progression of LV dysfunction and remodeling. ${ }^{10,11}$ Specifically, such compounds as metformin $^{21}$ and dichloroacetate (DCA) ${ }^{43}$ can improve glucose metabolism. Metformin acts by enhancing insulin sensitivity via the AMPK pathway. Studies have shown that metformin also attenuates cardiac fibrosis by inhibiting collagen synthesis. ${ }^{44}$ Moreover, studies in rats treated with dichloroacetate (DCA) showed increased glucose oxidation by attenuating increase in energy reserves, activation of the pentose phosphate pathway, and reduced oxidative stress improving cardiac function and animal survival. ${ }^{21}$ DCA has been used since its discovery in 1969 to treat lactic acidosis complications of congenital mitochondrial diseases and diabetes. It stimulates PDH and carbohydrate oxidation by inhibiting pyruvate dehydrogenase (PDH) kinase. Another drug (rapamycin) used for the prevention of transplant rejection inhibits mammalian target of rapamycin (mTOR) in SHRs, reducing cardiac hypertrophy but does not reduce blood pressure. ${ }^{45}$ Heart failure with preserved ejection fraction (HFpEF) is frequently accompanied by left ventricle hypertrophy and myocardial fibrosis. Histone deacetylases (HDACs) are a class of enzymes that cause conformation changes in the 3D architecture of DNA, modifying its transcription. HDAC inhibition with ITF2357 (givinostat) decreased hypertrophy and fibrosis and improved diastolic function in HFpEF patients. ${ }^{46}$ Other possible therapies for lipotoxicity in hearts of NAFLD patients can be learned from the diabetic heart. Wan and Rodrigues ${ }^{8}$ report on how the protein "ensemble" (heparanase-VEGF-LPL) cooperates in the diabetic heart to switch to predominantly use of fatty acid for energy; however, increased accumulation of fatty acid can trigger cell death by eliciting messengers such as ceramides. Understanding the interplay between heparanase, vascular endothelial growth factors (VEGFs), and LPL might help develop therapies for restoring metabolic equilibrium in cardiac lipotoxicity.

NAFLD is a serious disease with significant prognosis for cardiac death. The disease demonstrates abnormal structure and function in the liver cells that could potentially provide some insight into the same potential maladaptation of cardiac cells. The decrease in myocardial glucose uptake in NAFLD patients is a possible indicator of lipotoxicity in cardiac cells. Most PET studies reported in the literature involve using ${ }^{18} \mathrm{~F}$ FDG to assess cardiac glucose metabolism. However, the complex mechanisms of cardiac metabolism can only be appreciated using multiple imaging probes. PET imaging of metabolic processes has been predominately aimed at studying heart failure; however, PET imaging of cardiac fatty acid and glucose metabolism and perfusion in NAFLD patients would significantly help to direct therapeutic intervention and to prolong life for these patients. Dynamic cardiac PET can significantly help in the management of NAFLD by improving the diagnosis of cardiac disease.

\section{Disclosure}

Grant T. Gullberg receives financial compensation outside the submitted work from Spectrum Dynamics Medical, Inc. for consultancy and Solving Dynamics, Inc. for work performed on a small business grant from NIH and NSF. The other authors have no conflict of interest.

\section{References}

1. Correale M, Tarantino N, Petrucci R, Tricarico L, Laonigro I, Di Biase M, et al. Liver disease and heart failure: Back and forth. Eur J Intern Med 2018;48:25-34.

2. Lee HJ, Lee CH, Kim S, Hwang SY, Hong HC, Choi HY, et al. Association between vascular inflammation and non-alcoholic 
fatty liver disease: Analysis by ${ }^{18} \mathrm{~F}$-fluorodeoxyglucose positron emission tomography. Metabolism 2017;67:72-9.

3. Mahfood Haddad T, Hamdeh S, Kanmanthareddy A, Alla VM. Nonalcoholic fatty liver disease and the risk of clinical cardiovascular events: A systematic review and meta-analysis. Diabetes Metab Syndr 2017;11:S209-16.

4. Ong JP, Pitts A, Younossi ZM. Increased overall mortality and liver-related mortality in non-alcoholic fatty liver disease. J Hepatol 2008;49:608-12.

5. Eslam M, Valenti L, Romeo S. Genetics and epigenetics of NAFLD and NASH: Clinical impact. J Hepatol 2018;68:268-79.

6. Pais R, Giral P, Khan J-F, Rosenbaum D, Housset C, Poynard T, et al. Fatty liver is an independent predictor of early carotid atherosclerosis. J Hepatol 2016;65:95-102.

7. Abenavoli L, Peta V. Role of adipokines and cytokines in nonalcoholic fatty liver disease. Rev Recent Clin Trials 2014;9:13440

8. A Primer on Carbohydrate Metabolism in the Heart. In: Lopaschuk GD, Dhalla NS, editors. Cardiac energy metabolism in health and disease (advances in biochemistry in health and disease), 1st ed. New York; Springer; 2014.

9. Taegtmeyer H, Lam T, Davogustto G. Cardiac metabolism in perspective. Compr Physiol 2016;6:1675-99.

10. Stanley WC, Recchia FA, Lopaschuk GD. Myocardial substrate metabolism in the normal and failing heart. Physiol Rev 2005;85:1093-129.

11. Lopaschuk GD, Ussher JR, Folmes CDL, Jaswal JS, Stanley WC. Myocardial fatty acid metabolism in health and disease. Physiol Rev 2010;90:207-58.

12. Glatz JF, Luiken JJ, Bonen A. Membrane fatty acid transporters as regulators of lipid metabolism: Implications for metabolic disease. Physiol Rev 2010;90:367-417.

13. Nelson DL, Cox MM. Lehninger principles of biochemistry. 7th ed. New York, NY: W. H. Freeman and Company; 2017.

14. Peng KY, Watt MJ, Rensen S, Greve JW, Huynh K, Jayawardana $\mathrm{KS}$, et al. Mitochondrial dysfunction-related lipid changes occur in nonalcoholic fatty liver disease progression. J Lipid Res 2018;59:1977-86.

15. Schulze PC, Drosatos K, Goldberg IJ. Lipid use and misuse by the heart. Circ Res 2016;118:1736-51.

16. Schwenk RW, Holloway GP, Luiken JJFP, Bonen A, Glatz JFC. Fatty acid transport across the cell membrane: Regulation by fatty acid transporters. Prostaglandins Leukot Essential Fatty Acids 2010;82:149-54

17. Tang Y, Mi C, Liu J, Gao F, Long J. Compromised mitochondrial remodeling in compensatory hypertrophied myocardium of spontaneously hypertensive rat. Cardiovasc Pathol 2014;23:101-6.

18. Ikegami R, Shimizu I, Yoshida Y, Minamino T. Metabolomic analysis in heart failure. Circ J 2017;82:10-6.

19. Huang Y, Zhou M, Sun H, Wang Y. Branched-chain amino acid metabolism in heart disease: An epiphenomenon or a real culprit? Cardiovasc Res 2011;90:220-3.

20. Gropler RJ. Recent advances in metabolic imaging. J Nucl Cardiol 2013;20:1147-72.

21. Kundu BK, Zhong M, Sen S, Davogustto G, Keller SR, Taegtmeyer $\mathrm{H}$. Remodeling of glucose metabolism precedes pressure overload-induced left ventricular hypertrophy: Review of a hypothesis. Cardiology 2015;130:211-20.

22. Mather KJ, DeGrado TR. Imaging of myocardial fatty acid oxidation. Biochim Biophys Acta 2016;1861:1535-43.

23. Nesterov SV, Turta O, Han C, Mäki M, Lisinen I, Tuunanen H, et al. ${ }^{\mathrm{C}}-11$ acetate has excellent reproducibility for quantification of myocardial oxidative metabolism. Eur Heart $\mathrm{J}$ Cardiovasc Imaging 2015;16:500-6.
24. Wu KY, Dinculescu V, Renaud JM, Chen SY, Burwash IG, Mielniczuk LM, Beanlands RSB, deKemp RA. Repeatable and reproducible measurements of myocardial oxidative metabolism, blood flow and external efficiency using ${ }^{11} \mathrm{C}$-acetate PET. J Nucl Cardiol 2018 Feb 16.

25. Neely JR, Morgan HE. Relationship between carbohydrate and lipid metabolism and the energy balance of heart muscle. Ann Rev Physiol 1974;36:413-59.

26. Iozzo P, Bucci M, Roivainen A, Nagren K, Jarvisalo MJ, Kiss J, et al. Fatty acid metabolism in the liver, measured by positron emission tomography, is increased in obese individuals. Gastroenterology 2010;139:846-56.

27. Viljanen AP, Iozzo P, Borra R, Kankaanpaa M, Karmi A, Lautamaki R, et al. Effect of weight loss on liver free fatty acid uptake and hepatic insulin resistance. J Clin Endocrinol Metab 2009;94:50-5

28. Davila-Roman VG, Vedala G, Herrero P, de las Fuentes L, Rogers JG, Kelly DP, et al. Altered myocardial fatty acid and glucose metabolism in idiopathic dilated cardiomyopathy. J Am Coll Cardiol 2002;40:271-7.

29. De las Fuentes L, Herrero P, Peterson LR, Kelly DP, Gropler RJ, Davila-Roman VG. Myocardial fatty acid metabolism: independent predictor of left ventricular mass in hypertensive heart disease. Hypertension 2003;41:83-7.

30. Kudo T, Fukuchi K, Annala AJ, Chatziioannou AF, Allada V, Dahlbom $\mathrm{M}$, et al. Noninvasive measurement of myocardial activity concentrations and perfusion defect sizes in rats with a new small-animal positron emission tomograph. Circulation 2002;106:118-23

31. Taylor M, Wallhaus TR, DeGrado TR, Russell DC, Stanko P, Nickles RJ, et al. An evaluation of myocardial fatty acid and glucose uptake using PET with $\left[{ }^{18} \mathrm{~F}\right]$ fluoro-6-thia-heptadecanoic acid and $\left[{ }^{18} \mathrm{~F}\right] \mathrm{FDG}$ in patients with congestive heart failure. J Nucl Med 2001;42:55-62.

32. Wallhaus TR, Taylor M, DeGrado TR, Russell DC, Stanko P, Nickles RJ, et al. Myocardial free fatty acid and glucose use after carvedilol treatment in patients with congestive heart failure. Circulation 2001;103:2441-6.

33. Hernandez AM, Murphy ST, Zeng GL, Janabi M, Huber JS, Brennan KM, et al. Longitudinal evaluation of left ventricular substrate metabolism, perfusion, and dysfunction in the SHR model of hypertrophy using microPET imaging. J Nucl Med 2013;54:1938-45

34. Huber JS, Hernandez A, Janabi M, O'Neil JP, Brennan K, Murphy $\mathrm{S}$, et al. Longitudinal evaluation of myocardial fatty acid and glucose metabolism in fasted and nonfasted spontaneously hypertensive rats using microPET/CT. Mol Imaging 2017;16:1536012117724558.

35. Manabe O, Kikuchi T, Scholte AJHA, El Mahdiui M, Nishii R, Zhang M-R, et al. Radiopharmaceutical tracers for cardiac imaging. J Nucl Cardiol 2018;25:1204-36.

36. Li J, Lu J, Zhou Y. Mitochondrial-targeted molecular imaging in cardiac disease. BioMed Res Int 2017;2017:11.

37. Saraste A, Knuuti J. PET imaging in heart failure: The role of new tracers. Heart Fail Rev 2017;22:501-11.

38. Wey HY, Wang C, Schroeder FA, Logan J, Price JC, Hooker JM. Kinetic analysis and quantification of $\left[{ }^{11} \mathrm{C}\right]$ Martinostat for in vivo HDAC imaging of the brain. ACS Chem Neurosci 2015;6: 708-15.

39. Wu X, Wang P, Liu R, Zeng H, Chao F, Liu H, et al. Development of ${ }^{11} \mathrm{C}$-Labeled $\omega$-sulfhydryl fatty acid tracer for myocardial imaging with PET. Eur J Med Chem 2018;143:1657-66.

40. Li Y, Huang T, Zhang X, Zhong M, Walker NN, He J, et al. Determination of fatty acid metabolism with dynamic 
$\left[{ }^{11} \mathrm{C}\right]$ palmitate positron emission tomography of mouse heart in vivo. Mol Imaging 2015;14:516-25.

41. Shoup TM, Elmaleh DR, Bonab AA, Fischman AJ. Evaluation of trans-9- ${ }^{18}$ Ffluoro-3,4-Methyleneheptadecanoic acid as a PET tracer for myocardial fatty acid imaging. J Nucl Med 2005;46:297304.

42. Stone CK, Pooley RA, DeGrado TR, Renstrom B, Nickles RJ, Nellis SH, et al. Myocardial uptake of the fatty acid analog 14fluorine-18-fluoro-6-thia-heptadecanoic acid in comparison to beta-oxidation rates by tritiated palmitate. $\mathrm{J}$ Nucl Med 1998;39:1690-6.

43. Kato T, Niizuma S, Inuzuka Y, Kawashima T, Okuda J, Tamaki Y, et al. Analysis of metabolic remodeling in compensated left ventricular hypertrophy and heart failure. Circ Heart Fail 2010;3:42030
44. Yin M, van der Horst IC, van Melle JP, Qian C, van Gilst WH, Silljé $\mathrm{HH}$, et al. Metformin improves cardiac function in a nondiabetic rat model of post-MI heart failure. Am J Physiol Heart Circ Physiol 2011;301:H459-68.

45. Soesanto W, Lin HY, Hu E, Lefler S, Litwin SE, Sena S, et al. Mammalian target of rapamycin is a critical regulator of cardiac hypertrophy in spontaneously hypertensive rats. Hypertension 2009;54:1321-7.

46. Jeong MY, Lin YH, Wennersten SA, Demos-Davies KM, Cavasin MA, Mahaffey JH, et al. Histone deacetylase activity governs diastolic dysfunction through a nongenomic mechanism. Sci Transl Med 2018;10:eaaao0144. 